\title{
FVM-Modeling of Continuity-Coupled Electrical Charge Submitted to Incompressible Flow with OpenFOAM®
}

\author{
$\begin{array}{lll}\text { D. Rubinetti } & & \text { D. A. Weiss } \\ & 1\end{array}$ \\ ${ }^{1}$ Institute of Thermal- and Fluid-Engineering , University of Applied Sciences and Arts Northwestern Switzerland
}

\begin{abstract}
This study conceives a FVM-based model for ionization processes coupling the incompressible Navier-Stokes equations to Maxwell's equations adjusted for electrostatics. Modeling instructions for the opensource tool OpenFOAM $^{\mathrm{TM}}$ are presented within the scope of defining a customized solver which calculates the distortion of electrical current by an external fluid flow.

By means of a simplified test-case the neglegibile impact of external convection on the distribution of space charge for many engineering applications is confirmed while pointing out the limitations of negligible convection. The study shows that peak velocities beyond 100 $\mathrm{m} \mathrm{s}^{-1}$ within the flow field might have an impact on the current distribution, thus requiring careful evaluation of the modeling assumptions.

The approach described in the modeling guide proves to be numerically stable. The results for the electric field distribution have been analytically verified.
\end{abstract}

Keywords: Corona Discharge, Fluid Flow, Electrodynamics, Space Charge Density, Convection

\section{Introduction}

Corona discharge describes the effect where a nonconducting gaseous carrier such as air is ionized in proximity of an electrode with strong electrical field. On molecular level the air molecule receives electrons from the electrode (negative polarity) or loses electrons to the electrode (positive polarity). In either case the ionized molecule is accelerated away from the electrode towards the grounded electrode. The sum of all ionized molecules traveling approximately on electric field lines are described as a continuum and as such it is subject to macroscopic transport phenomena. Ionization processes are widely used i.e. to reduce the environmental impact of combustion-based pollution. To assist the design of such applications numerical models prove to be a costeffective and powerful tool. Among numerous considerations for the numerical modeling of Corona discharge and ionization processes as such, the assumption of negligible external convection is prevalent. This study tackles the widespread convention of excluding convection by means of a geometrically simplified test-case run by a customized solver which includes fluid dynamics and electrostatics in a coupled way.

\section{NOMENCLATURE}

\begin{tabular}{lll}
\hline$b$ & ion mobility & $\mathrm{m}^{2} \mathrm{~V}^{-1} \mathrm{~s}^{-1}$ \\
$E_{0}$ & Corona onset field strength & $\mathrm{V} \mathrm{m}^{-1}$ \\
$\hat{E}$ & dimensionless electric field & - \\
$f_{r}$ & fatigue factor & - \\
$g$ & gravitational acceleration & $\mathrm{m} \mathrm{s}^{-2}$ \\
$I$ & electric current per unit length & $\mathrm{A} \mathrm{m}^{-1}$ \\
$\hat{I}$ & dimensionless electric current & - \\
$j$ & current density & $\mathrm{Am}^{-2}$ \\
$m_{s}$ & relative gas density & - \\
$r$ & radius & $\mathrm{m}$ \\
$r_{1}$ & charging electrode radius & $\mathrm{m}$ \\
$r_{2}$ & ground electrode radius & $\mathrm{m}$ \\
$p$ & pressure & $\mathrm{Pa}$ \\
$p_{0}$ & standard pressure & $\mathrm{Pa}^{-}$ \\
$T_{0}$ & standard temperature & $\mathrm{K}$ \\
$u$ & fluid velocity field & $\mathrm{m} \mathrm{s}^{-1}$ \\
$u_{r}$ & radial fluid velocity & $\mathrm{m} \mathrm{s}^{-1}$ \\
$V$ & volumetric flow per unit length & $\mathrm{m}^{2} \mathrm{~s}^{-1}$ \\
$\hat{V}$ & dimensionless volumetric flow & - \\
$\delta$ & boundary cell layer height & $\mathrm{m}^{-1}$ \\
$\varepsilon_{0}$ & vacuum permittivity & $\mathrm{Fm}^{-1}$ \\
$\varepsilon_{r}$ & relative permittivity & - \\
$v$ & kinematic viscosity & $\mathrm{m}^{2} \mathrm{~s}^{-1}$ \\
$\phi$ & electric potential & $\mathrm{kV}^{-3}$ \\
$\rho_{e l}$ & space charge density & $\mathrm{Cm}$ \\
\hline & & \\
\hline
\end{tabular}




\section{Physical Model}

The physical nature of electrical charge in a gaseous environment is best explained by electrostatic precipitation a widely used application area for the control of airborne particle emissions. In practice, a high-voltage electrode (typically $10-50 \mathrm{kV}$ ) is immersed in exhaust gases from i.e. cement plants. By ionizing the surrounding air, soot particles are being electrically charged and accelerated out of the main exhaust gas flow. Thus, cleaning the gas from toxic components. Figure 1 illustrates the multiphysical coupling of such systems.

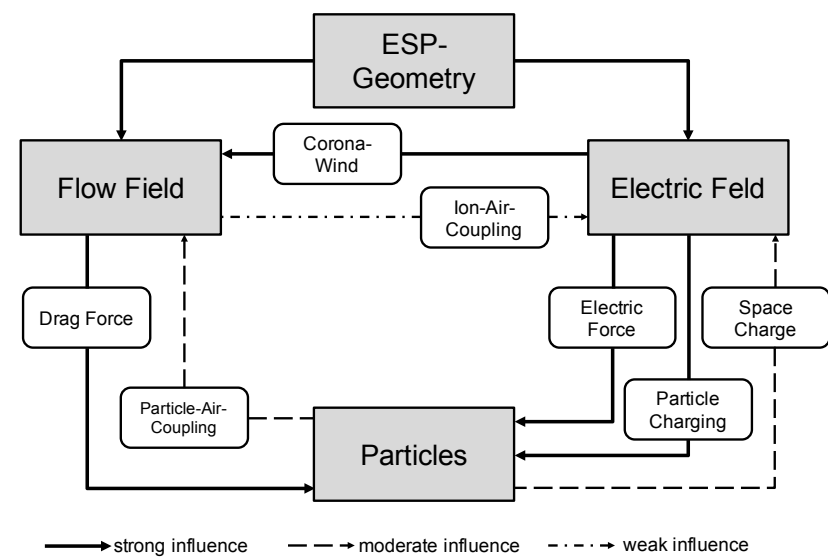

Figure 1. Multiphysics coupling of electrostatic precipiators (Rubinetti et al., 2017)

In this study the interdependence of flow field and electric field is studied. The production and acceleration of air molecules leads to a strong influence of the electric field onto the flow field via Corona wind. Hereby, an initially stagnating fluid $(u=0 \mathrm{~m} / \mathrm{s})$ undergoes an acceleration itself. The reverse effect, when the flow field distorts the propagation of ions, is often neglected due to its assumed weak influence (Rubinetti et al., 2015). As a characteristic scale on molecular level it can be stated that ions move with a velocity of $\sim 1000 \mathrm{~m} \mathrm{~s}^{-1}$ on electric field lines while the surrounding fluid in most applications has a significantly lower characteristic velocity.

The study is carried out with two coaxially arranged cylinders of $r_{1}=0.002 \mathrm{~m}$ and $r_{2}=0.2 \mathrm{~m}$ as shown in figure 2 . Hereby the inner cylinder represents the wire as the charging electrode and the outer cylinder is the grounded electrode. The coaxial geometry is reduced to a quarter of a cylinder slice with one mesh cell in axial direction, thus scaling the problem from 3D to 2D suppressing alterations in axial direction.

Table 1 lists the assumptions and definitions used in the study. In this theoretical case the charging electrode $\partial \Omega_{1}$ is assumed to carry a given space charge density for the electrostatic part and simultaneously act as inlet for the fluid flow part with a velocity of $w=100 \mathrm{~m} \mathrm{~s}^{-1}$ radially distributed.

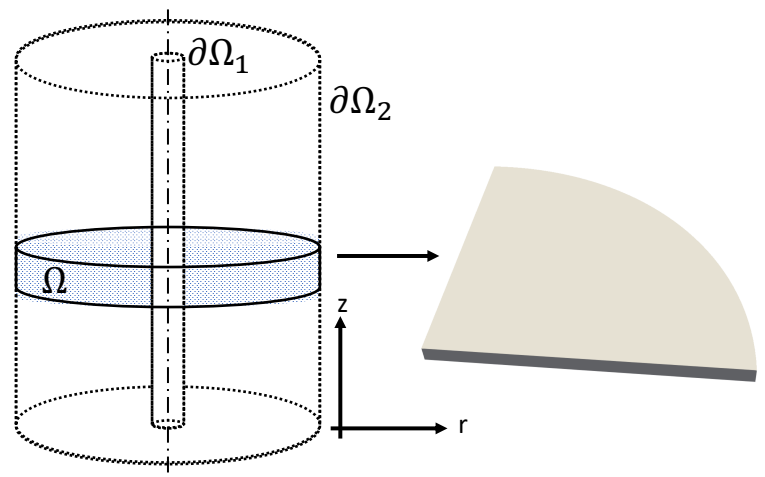

Figure 2. Simplified coaxial geometry

The Corona onset field strength in $\left[\mathrm{V} \mathrm{m}^{-1}\right]$ is given by the empirical correlation (White, 1963)

$$
E_{0}=3 \times 10^{6} f_{r}\left(m_{s}+0.03 \sqrt{\frac{m_{s}}{r_{1} / 1[\mathrm{~m}]}}\right)
$$

with the fatigue factor $f_{r}$ being a measure for the usage of the electrode ( $f_{r}=0.6$ for practical use, $f_{r}=1$ for a new electrode). The relative gas density takes into account the surrounding fluid, which, at standard conditions $\left(T_{0}, p_{0}\right)$ has the value of $m_{s}=1$.

\section{Numerical Model}

The numerical model considers the incompressible Navier-Stokes equations and Maxwells equations for electrostatic applications without magnetic influence. A modelling approach for the definition of a customized solver for OpenFOAM ${ }^{\circledR}$ is given.

\subsection{Governing equations}

For the fluid flow part the continuity equation yields

$$
\nabla \cdot \boldsymbol{u}=0
$$

while the incompressible Navier-Stokes equation writes

$$
\frac{\partial \boldsymbol{u}}{\partial t}+(\boldsymbol{u} \cdot \nabla) \boldsymbol{u}-v \nabla^{2} \boldsymbol{u}=-\frac{1}{\rho} \nabla p+\boldsymbol{g} .
$$

As for the electrostatics part, neglecting magnetic influences in Maxwells equations, the electric field can be expressed in terms of the gradient of the electric potential

$$
\boldsymbol{E}=-\nabla \phi
$$

which leads us to the Poisson equation for electric potential with a source term including the space charge density $\rho_{e l}$

$$
\nabla^{2} \phi=-\frac{\rho_{e l}}{\varepsilon_{0}} .
$$

Equations (4) and (5) are subject to the conservation of electrical chargs such that 
Table 1. Definition of model parameters

\begin{tabular}{lccc}
\hline & Charging Electrode & Grounded Electrode & Domain \\
& $\partial \Omega_{1}$ & $\partial \Omega_{2}$ & $\Omega$ \\
\hline Radius $r[\mathrm{~m}]$ & 0.002 & 0.2 & \\
Electric potential $\phi[\mathrm{kV}]$ & 50 & 0 & \\
Space charge density $\rho_{e l}\left[\mathrm{Cm}^{-3}\right]$ & 0.001 & - & \\
Fluid velocity $u_{r}\left[\mathrm{~m} \mathrm{~s}^{-1}\right]$ & 50 & - & \\
Corona onset field strength $E_{0}\left[\mathrm{~V} \mathrm{~m}^{-1}\right]$ & $5.01 \times 10^{6}$ & & 1 \\
Fatigue factor $f_{r}$ & 1 & & 0.001 \\
Relative gas density $m_{s}[-]$ & & & 1 \\
Ion mobility $b\left[\mathrm{~m}^{2} \mathrm{~V}^{-1} \mathrm{~s}^{-1}\right]$ & & & 298.15 \\
Vacuum permittivity $\varepsilon_{0}\left[\mathrm{Fm} \mathrm{F}^{-1}\right]$ & & & 1 \\
Relative permittivity $\varepsilon_{r}[-]$ & & & \\
Standard temperature $T_{0}[\mathrm{~K}]$ & & & \\
Standard pressure $p_{0}[\mathrm{~atm}]$ & & & \\
\hline
\end{tabular}

$$
\frac{\partial \rho_{e l}}{\partial t}+\nabla \cdot \boldsymbol{j}=0
$$

While the current density $\boldsymbol{j}$ is given by

$$
\boldsymbol{j}=\rho_{e l}(\boldsymbol{u}+b \boldsymbol{E})
$$

which forms the core of the present investigation due to its convective part $\rho_{e l} \boldsymbol{u}$ which is often neglected alongside with diffusion.

\subsection{Implementation}

The following description points at implementing electrostatics into OpenFOAM ${ }^{\circledR}$ syntax. The costumization of the solver can be achieved by compiling the built-in icoFoam structure with additional lines of code for the electrostatics part. Firstly, the governing equation for Poissons equation (5)

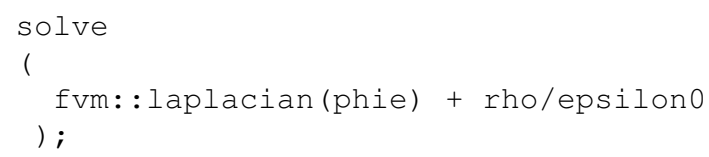

with a newly defined variable phie for the electric potential. Second point in the iteration procedure is the convective transport of the space charge, as $b \boldsymbol{E}+\boldsymbol{u}$ as seen in equation (7)

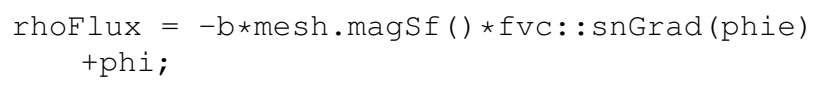

where phi accounts for the fluid-flow velocity field $\boldsymbol{u}$. The variable phi is pre-defined by icoFoam and shall not be confused with the newly defined variable for the electric field phie. Finally, to ensure charge conservation as given by equation (6) the following step shall be added as

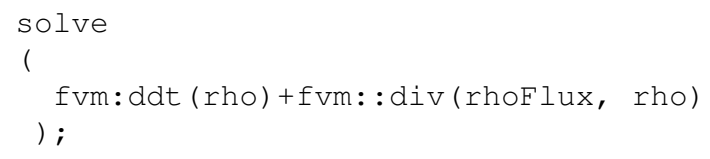

For postprocessing purposes it proves useful to include an optional command which writes a separate solution file for the electric field, that is

$$
\text { Efield == -fvc: :grad(phie); }
$$

To write out an additional solution file to the electric field changes must be included also in the createFields. $H$ file. Moreover, in the "0" folder an Efield file must be specified. Because the value of the electric field is determined by the solution variable for the electric potential, it is recommended not to override the value of the electric field at the boundaries of the electrode. Instead, the boundary condition extrapolatedCalculated in the electrical field file for both electrode boundaries shall be used to ensure that the electrical field variable is written out after and based on the electric potential variable during each iteration taken by the solver.

\subsection{Mesh}

For the calculations a blockmesh is used with 150 cells in radial direction and 80 cells in azimutal direction, while one cell is present in axial direction. The radial distribution of the cells is set with simpleGrading (400), resulting in a first cell width to radius ratio of 0.02 according to figure 3 . The high resolution of the radial component in proximity of the charging electrode is needed to ensure the correct solution of high gradients of the electric field.

The total number of cells amounts to 12'000 Finite Volumina which on a $2.6 \mathrm{GHz}$ CPU with $8 \mathrm{~GB}$ RAM require 244 seconds of CPU runtime. The simulation time goes from $0 \mathrm{~s}$ to $0.05 \mathrm{~s}$ with a timestep of $5 \times 10^{-6} \mathrm{~s}$.

\section{Analytical Verification}

The coaxial cylinder test-case geometry allows the formulation of an analytical solution for the electric field. As no changes in axial direction are expected, the volumetric flow per unit length can be expressed in terms of the radial velocity as: 


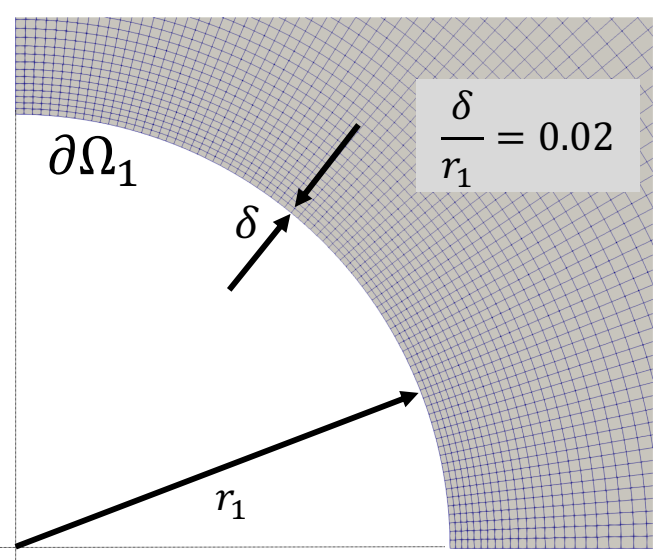

Figure 3. Cutout of the mesh used in the calculations with focus on the charging electrode

$$
V=2 \pi r u_{r}
$$

whereas the electric current is quantified in a similar way including the current density in radial direction

$$
I=2 \pi r j_{r}=2 \pi r\left(\rho_{e l}\left(u_{r}+b E_{r}\right)\right)
$$

To work around integration constants, the volumetric flow and electric current are non-dimensionalized using the quantities listed in table 1 as

$$
\begin{gathered}
\hat{V}=\frac{V}{2 \pi b r_{1} E_{0}} \\
\hat{I}=\frac{I}{2 \pi \varepsilon_{0} \varepsilon_{r} b E_{0}^{2}}
\end{gathered}
$$

With the non-dimensional radius $\hat{r}=r / r_{1}$ the dimensionless electric field takes the form

$$
\hat{E}=\frac{-\hat{V} \pm \sqrt{\hat{I}\left(\hat{r}^{2}-1\right)(\hat{V}+1)^{2}}}{\hat{r}}
$$

for the case of positive convection, that is, when the electric current and fluid flow have the same direction. In countercurrent the term $(\hat{V}+1)^{2}$ becomes $(\hat{V}-1)^{2}$.

\section{Results}

\subsection{Velocity field}

The velocity field is shown in figure 4 with unscaled velocity vectors. As expected, the flow starts from the electrode with an intensity of $50 \mathrm{~ms}^{-1}$. Radially, the velocity decreases rapidly, due to large spatial increase and dispersion.

\subsection{Electric field}

The resulting electric field is shown in figure 5 and figure 6. It can be seen that a fine mesh resolution around the charging electrode is necessary to reliably represent the sharp gradients of the electric field.

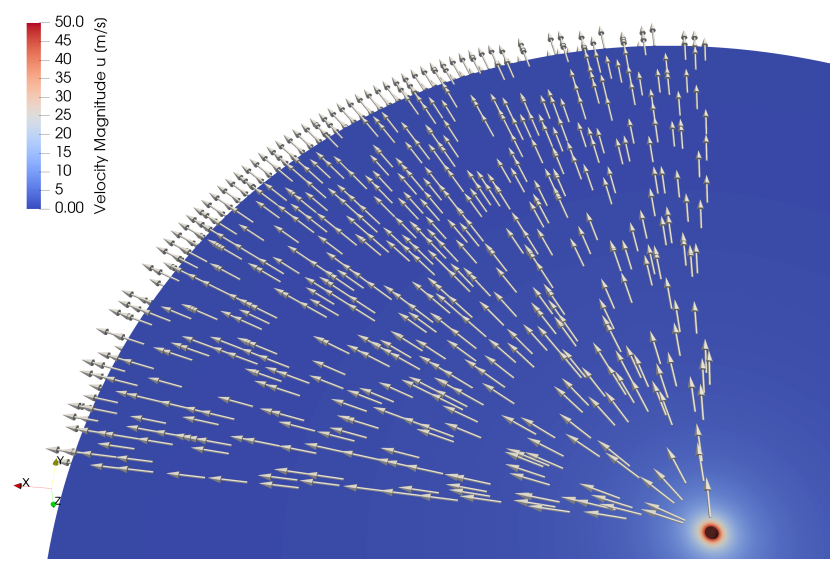

Figure 4. Visualization of the velocity field

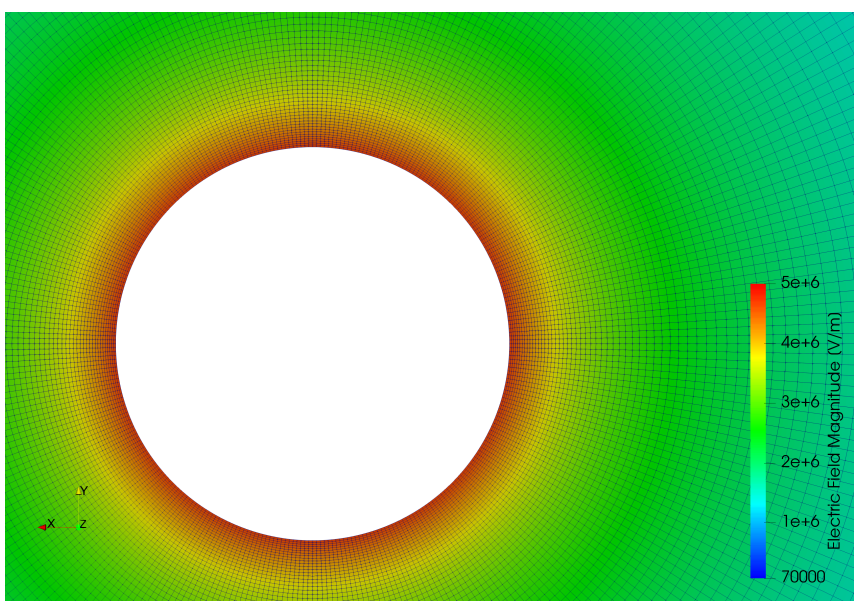

Figure 5. Close-up view of the electric field

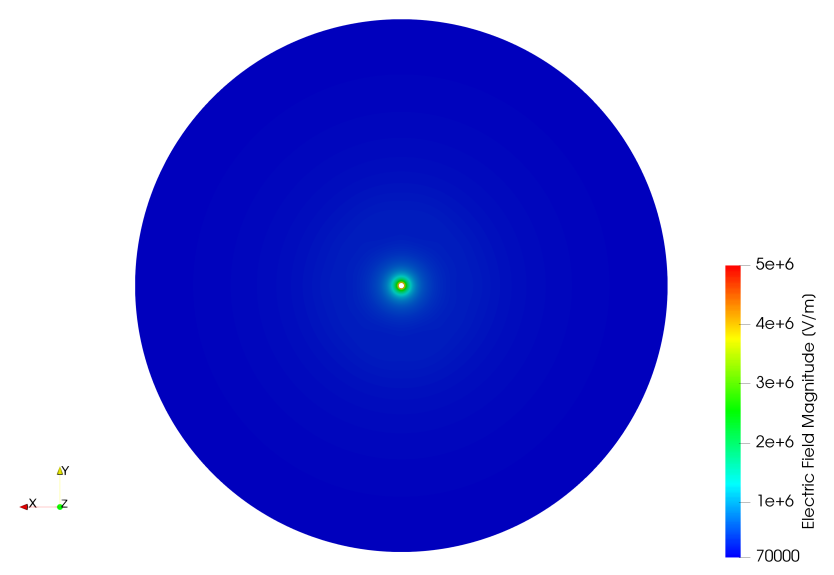

Figure 6. Global view of the electric field 
For the given setup the solution for the radial distribution of the coaxial geometry is analytically verified as shown in figure 7.

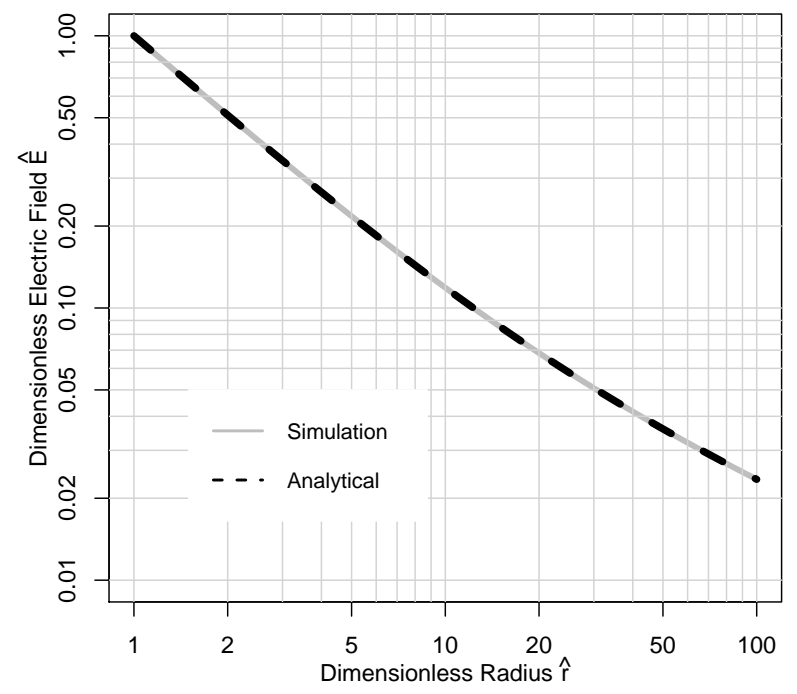

Figure 7. Verification of the electrical field along the radius

\subsection{Discussion}

In this hypothetical test-case the results for the electrostatics are analytically verified which consequently confirms the validity of the presented modeling procedure. For this geometry with the given model parameters for air there is no noticeable influence of convection. In many engineering applications where ionization processes are part of a larger construct the assumption of negligible convection holds.

However, as illustrated by the sensitivity analysis in figure 8 under certain flow patterns and regimes the influence of convection might be of considerable importance, i.e. for supersonic velocities the electric field experiences an increase further away from the electrode. In practice, this effect can be understood as deviation of current, where to the most extreme extent it could be possible to reverse the direction of electrical current flow merely by external convection.

For the test-case and the verification of the modeling strategy a constant value for the initial space charge density $\rho_{e l}$ is appropriate. Future work will target a more complex FVM modeling methodology aiming at dynamically computing the value of the space charge density within the interations taken by the solver. The interested reader may be referred to (Rubinetti et al., 2017) for an FEM-based approach.

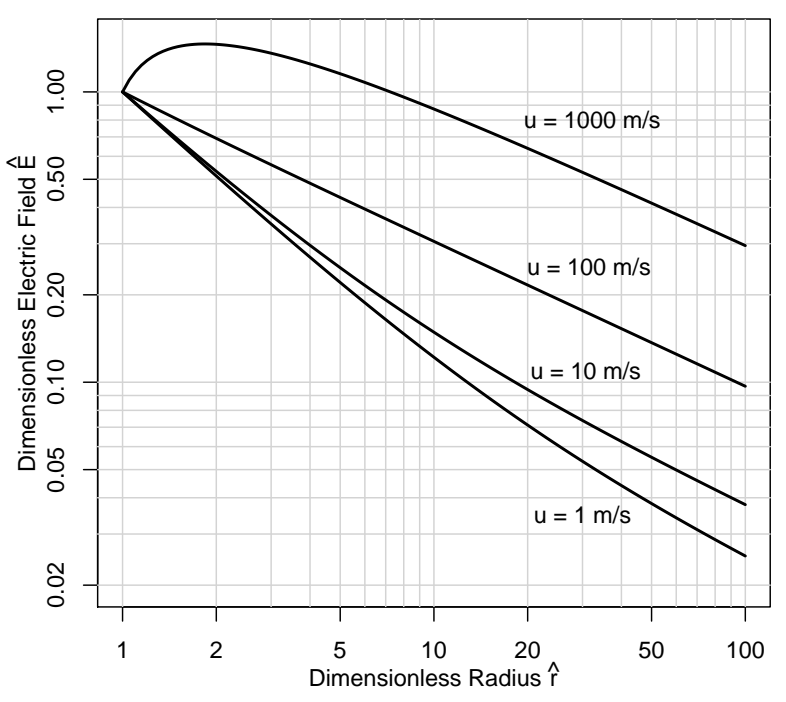

Figure 8. Sensitivity analysis for the electric field with different velocities assuming ion mobility $b=10^{-6} \mathrm{~m}^{2} \mathrm{~V}^{-1} \mathrm{~s}^{-1}$

\section{Conclusion}

The presented test-case and the corresponding modeling methodology for OpenFOAM® is numerically stable and shows the suitability of FVM for the multiphysical coupling of electrostatics and fluid dynamics. For the case of air ionization and an overlaying velocity field with $50 \mathrm{~m} \mathrm{~s}^{-1}$ peak velocity convection has no noticeable influence - thus confirming the wide-spread modeling assumption of negligible external convection for most engineering applications involving Corona discharge.

The results have been analytically verified including a sensitivity analysis showing that for certain flow regimes convection might need to be considered to ensure physical accuracy.

\section{Acknowledgements}

Gernot Boiger and Dirk Wilhelm are gratefully acknowledged for the fruitful discussions conducted during the development of the customized OpenFOAM ${ }^{\circledR}$ solver in this work.

\section{References}

Ulrich Kogelschatz, Walter Egli, and Edgar A. Gerteisen. Advanced computational tools for electrostatic precipitators. $A B B$ review, (4):33-42, 1999.

Donato Rubinetti, Daniel A Weiss, and Walter Egli. Electrostatic precipitators-modelling and analytical verification concept. In University of Applied Sciences Northwestern Switzerland, Windisch, Switzerland, COMSOL CONFERENCE, 2015.

Donato Rubinetti, Daniel A. Weiss, and Walter Egli. Corona 
discharge-a fully coupled numerical approach verified and validated. International Journal of Multiphysics, 11(4), 2017.

Harry J. White. Industrial electrostatic precipitation. In Industrial electrostatic precipitation. Addison-Wesley, 1963. 